\title{
ATIYAH-PATODI-SINGER BOUNDARY CONDITION AND A SPLITTING FORMULA OF A SPECTRAL FLOW
}

\author{
KENRO FURUTANI
}

\begin{abstract}
We describe a relation between Atiyah-Patodi-Singer boundary condition and a global elliptic boundary condition which naturally appears in formulating a splitting formula for a spectral flow, when we decompose the manifold into two components. Then we give a variant of the splitting formula with the Hörmander index as a correction term.
\end{abstract}

\section{Contents}

\begin{tabular}{|lr|}
\hline 1. Introduction & 1 \\
\hline 2. A global elliptic boundarv condition & 2 \\
\hline 3. Svmplectic reduction theorem & 5 \\
\hline 4. Cauchy data spaces and Hörmander index & 7 \\
\hline 5. A splitting formula of a spectral flow & 8 \\
\hline References & 10 \\
\hline
\end{tabular}

\section{INTRODUCTION}

In the paper [FO] we formulated and proved a splitting formula of a spectral flow for a continuous family of first order selfadjoint elliptic differential operators $\left\{A_{t}\right\}_{t \in[0,1]}$ defined on a closed manifold $M$. This is an addition formula of a spectral flow when we decompose a manifold into two components along a hypersurface $\Sigma, M=M_{-} \cup_{\Sigma} M_{+}$, $\partial M_{ \pm}=\Sigma$. For such a family $\left\{A_{t}\right\}$ considered on the whole closed manifold $M$, an integer, called spectral flow, is well defined, and to "observe" this quantity we cut the manifold along a hypersurface, then we can "observe" from the hypersurface a quantity "Maslov index" which is a curve of boundary data of solutions of operators. This quantity can be understood as the spectral flow. This is just a spectral flow formula ([Yo], [Ni]) where manifolds need not be separated into two components. If the manifold is separated into two parts by the hypersurface, then we will have two Maslov indexes which together give the whole information of the spectral flow. For this observation we must make clear which family we are observing, i.e., to get a family of selfadjoint Fredholm extensions we must impose a suitable elliptic boundary condition on the family $\left\{A_{t}\right\}$ when we restrict the operators on each component $M_{ \pm}$. This condition appears in a natural way in our formulation to write down the splitting formula and reflects the influence from one side to other side. On the other hand Atiyah-Patodi-Singer boundary condition is described

2000 Mathematics Subject Classification. 58J30, 58J32, 53D12.

Key words and phrases. spectral flow, Maslov index, Atiyah-Patodi-Singer boundary condition, Hörmander index, Fredholm-Lagrangian-Grassmannian, elliptic boundary condition. 
based on the boundary data. Nevertheless these two are relating each other in the case of the operators of the product form near the hypersurface.

The purpose of this paper is to describe a relation between our global elliptic boundary condition and Atiyah-Patodi-Singer boundary condition. There are several such splitting formulas ([CLM2], [DK], [KL], [Ta], [Ni] ) and the boundary condition treated there is mostly Atiyah-Patodi-Singer boundary condition. So the result in this paper will give us a sight of our global elliptic boundary condition for operators satisfying suitable analytic assumptions.

We follow the theory of symplectic Hilbert spaces, especially of the Fredholm-LagrangianGrassmannian and the Maslov index in the infinite dimension which were discussed in the papers [Fu] and [FO] precisely(also see [Go, [CLM1] and [RS]).

In $\S 2$ we explain a global elliptic boundary condition appearing in the splitting formula for a spectral flow and state a relation between it and Atiyah-Patodi-Singer boundary condition in terms of the Fredholm-Lagrangian-Grassmannian.

In $\S 3$, first we recall a $L_{2}$-reduction theorem [Fu and by applying this we give a proof of Theorem (2.7).

In $\S 4$ as an application of Theorem (2.7) we give a variant of a splitting formula of a spectral flow with Hörmander index as a correction term.

\section{A gLOBAl ELLIPTIC BOUNDARY CONDITION}

In this section we explain an elliptic boundary condition we introduced in [FO].

Let $A$ be a first order selfadjoint elliptic differential operator defined on a real vector bundle $\mathbb{E}$ on a closed manifold $M$. Let $\Sigma$ be a hypersurface of $M$ along which $M$ is separated into two components $M_{ \pm}, M=M_{-} \cup_{\Sigma} M_{+}, \partial M_{ \pm}=\Sigma$, and we denote the first order Sobolev space on $M$ (resp. $M_{ \pm}$) taking values in the real vector bundle $\mathbb{E}$ by $H^{1}(M, \mathbb{E})\left(\right.$ resp. $\left.H^{1}\left(M_{ \pm}, \mathbb{E}_{\mid M_{ \pm}}\right)\right)$. For the subspace in $H^{1}\left(M_{ \pm}, \mathbb{E}_{\mid M_{ \pm}}\right)$with vanishing boundary values we denote it by $H_{0}^{1}\left(M_{ \pm}, \mathbb{E}_{\mid M_{ \pm}}\right)$. These are the domains of the minimal closed extensions of the operators $A$ considered on $C_{0}^{\infty}\left(M_{ \pm} \backslash \Sigma, \mathbb{E}_{\mid M_{ \pm} \backslash \Sigma}\right)$ and we denote them by $\mathfrak{D}_{\mathbf{m}}^{ \pm}=H_{0}^{1}\left(M_{ \pm}, \mathbb{E}_{\mid M_{ \pm}}\right)$. Then we denote by $\mathcal{A}_{ \pm}^{*}$ their adjoint operators considered on $M_{ \pm}$and by $\mathfrak{D}_{\mathbf{M}}^{ \pm}$their domains of definitions, i.e., $f \in \mathfrak{D}_{\mathbf{M}}^{ \pm}$if $f \in L_{2}\left(M_{ \pm}, \mathbb{E}_{\mid M_{ \pm}}\right)$and $A(f) \in L_{2}\left(M_{ \pm}, \mathbb{E}_{\mid M_{ \pm}}\right)$in the distribution sense.

We must put two assumptions (a1) and (a2) on the selfadjoint elliptic operator $A$ :

(a1): A satisfies the unique continuation property with respect to the hypersurface $\Sigma$, that is,

$$
\operatorname{Ker}\left(\mathcal{A}_{ \pm}^{*}\right) \cap \mathfrak{D}_{\mathbf{m}}^{ \pm}=\{0\} .
$$

(a2): On a tubular neighborhood $\mathcal{N} \cong(-1,1) \times \Sigma$ the operator $A$ is of the product form, that is,

$$
A=\sigma\left(\frac{\partial}{\partial u}+B_{0}\right)
$$

where $\sigma$ is a bundle map on $\mathbb{E}_{\mid \mathcal{N}}$ which does not depend on the normal variable $u \in(-1,1)$, the operator $B_{0}$ is a selfadjoint elliptic operator on $\Sigma$ and also does not depend on the normal variable $u$.

We identify $\mathcal{N} \cap M_{-} \cong(-1,0] \times \Sigma$ and $\mathcal{N} \cap M_{+} \cong[0,1) \times \Sigma$. 
The selfadjointness of the operator $A$ implies that

$$
\begin{aligned}
& \sigma^{2}=-\mathrm{Id},{ }^{t} \sigma=-\sigma \\
& \sigma \circ B_{0}+B_{0} \circ \sigma=0,
\end{aligned}
$$

where the transpose ${ }^{t} \sigma$ is taken with respect to a suitable metric on the vector bundle $\mathbb{E}$. We denote the inner product on the $L_{2}$ sections of $\mathbb{E}$ by $<\cdot, \cdot>$. Then $\sigma$ defines an almost complex structure and a compatible symplectic structure on $L_{2}\left(\Sigma, \mathbb{E}_{\mid \Sigma)}\right)=L_{2}(\Sigma)$.

Let $\mathfrak{D}_{0}$ be a subspace in $H^{1}\left(M_{-}, \mathbb{E}_{\mid M_{-}}\right)$defined by

$\mathfrak{D}_{0}=\left\{\left.f \in H^{1}\left(M_{-}, \mathbb{E}_{\mid M_{-}}\right)\right|^{\exists} \tilde{f} \in H^{1}(M, \mathbb{E})\right.$ such that $\tilde{f}_{\mid M_{-}}=f$, and $A(f)=0$ on $\left.M_{+}\right\}$.

Hereafter we will state properties only for the case $M_{-}$, but shall use the corresponding results for $M_{+}$, if necessary.

We denote the restriction of $\mathcal{A}_{-}^{*}$ to $\mathfrak{D}_{0}$ by $A_{\mathfrak{D}_{0}}$, then under the two assumptions (a1) and (a2) we have

Proposition 2.1. The operator $A_{\mathfrak{D}_{0}}$ satisfies the inequality:

$$
\|f\|_{1} \leq c\left(\left\|A_{\mathfrak{D}_{0}}(f)\right\|+\|f\|\right), \forall f \in \mathfrak{D}_{0}
$$

with a positive constant $c>0$, where $\|f\|_{1}$ denotes the first order Sobolev norm. And so $A_{\mathfrak{D}_{0}}$ is selfadjoint and has compact resolvents.

This property is basic to state Theorem (2.7) below. We already made use of this property in our paper ([F] $)$. The proof is given upon $L_{2}$-reduction theorem of the Maslov index in the infinite dimension. In the next section we explain some part of a proof of this proposition, and together with the help of Rellich's Theorem we prove Theorem (2.7).

Remark 2.2. If $A$ is invertible on $H^{1}(M, \mathbb{E})$, then the same holds for $A_{\mathfrak{D}_{0}}$ as in the above proposition without assumptions (a1) and (a2), because we have

$\|f\|_{H^{1}\left(M_{-}, \mathbb{E}_{M_{-}}\right)} \leq C\left(\|f\|_{H^{1}(M, \mathbb{E})}+\|f\|_{L_{2}(M)}\right)=C\left(\|f\|_{H^{1}\left(M_{-}, \mathbb{E}_{\mid M_{-}}\right)}+\|f\|_{L_{2}(M)}\right) \leq C^{\prime}\|f\|_{H^{1}\left(M_{-}, \mathbb{E}_{\mid M_{-}}\right)}$

for $f \in H^{1}(M, \mathbb{E})$ satisfying $A(f)=0$ on $M_{+}$.

Let $\left\{\ell_{k}\right\}_{k \in \mathbb{Z} \backslash\{0\}}$ be the eigenvalues of the operator $B_{0}$ and we denote corresponding orthonormal eigensections by $\left\{\varphi_{k}\right\}$. From the properties (2.3) and (2.4) we have $\ell_{k}=$ $-\ell_{-k}>0$ for $k=N_{0}+1, N_{0}+2, \cdots$ with $N_{0}=1 / 2 \cdot \operatorname{dim} \operatorname{Ker}\left(B_{0}\right)$ (see Remark (2.4) below) and $\ell_{k}=0$ for $0<|k| \leq N_{0}$.

For a section $\varphi$ on $\Sigma$ let

$$
\varphi=\sum_{k \in \mathbb{Z} \backslash\{0\}} a_{k} \varphi_{k}
$$

be the eigensection-expansion, then the Sobolev space $H^{s}\left(\Sigma, \mathbb{E}_{\mid \Sigma}\right)$ of order $s \in \mathbb{R}$ on $\Sigma$ is characterized as

$$
H^{s}\left(\Sigma, \mathbb{E}_{\mid \Sigma}\right)=\left\{\varphi=\left.\sum_{k \in \mathbb{Z} \backslash\{0\}} a_{k} \varphi_{k}\left|\sum_{k \in \mathbb{Z} \backslash\{0\}}\right| a_{k}\right|^{2}\left|\ell_{k}\right|^{2 s}<\infty\right\} .
$$

Let $\mathfrak{D}_{A P S}^{0}$ be a subspace in $H^{1}\left(M_{-}, \mathbb{E}_{\mid M_{-}}\right)$such that

$$
\begin{aligned}
& \mathfrak{D}_{A P S}^{0} \\
& =\left\{f \in H^{1}\left(M_{-}, \mathbb{E}_{\mid M_{-}}\right) \mid \text {if } f_{\mid \Sigma}=\sum_{k \in \mathbb{Z} \backslash\{0\}} a_{k} \varphi_{k}, \text { then } a_{-k}=0 \text { for } k=1,2,3, \cdots\right\},
\end{aligned}
$$


and we denote the restriction of $\mathcal{A}_{-}^{*}$ to $\mathfrak{D}_{A P S}^{0}$ by $A_{\mathfrak{D}_{A P S}^{0}}$. This non-local boundary condition (2.6) is called "Atiyah-Patodi-Singer boundary condition". Then

Proposition 2.3. ([APS] ) The operator $A_{\mathfrak{D}_{A P S}^{0}}$ defined on $\mathfrak{D}_{A P S}^{0}$ is a selfadjoint operator with compact resolvents.

Now let $\beta^{-}=\mathfrak{D}_{\mathbf{M}}^{-} / \mathfrak{D}_{\mathbf{m}}^{-}$be the space of boundary values. Here the maximal domain $\mathfrak{D}_{\mathbf{M}}^{-}$is equipped with the norm $\|\cdot\|^{G}$ given by the graph inner product:

$$
<f, g>^{G}=<f, g>+<\mathcal{A}_{-}^{*}(f), \mathcal{A}_{-}^{*}(g)>.
$$

The space $\beta^{-}$has a structure of a symplectic Hilbert space with the symplectic form

$$
\omega([f],[g])=<\mathcal{A}_{-}^{*}(f), g>-<f, \mathcal{A}_{-}^{*}(g)>, f, g \in \mathfrak{D}_{M}^{-},
$$

and is realized in the distribution space on $\Sigma$ :

$$
\begin{aligned}
& \beta^{-}=\left\{f \in H^{-1 / 2}\left(\Sigma, \mathbb{E}_{\mid \Sigma}\right) \mid f=\sum_{k \in \mathbb{Z} \backslash\{0\}} c_{k} \varphi_{k}, \text { with } \sum_{k>0}\left|c_{k}\right|^{2} \ell_{k}<\infty\right. \text { and } \\
& \left.\sum_{k<0}\left|c_{k}\right|^{2}\left|\ell_{k}\right|^{-1}<\infty\right\} \\
& =\left\{f \in H^{1 / 2}\left(\Sigma, \mathbb{E}_{\mid \Sigma}\right) \mid f=\sum_{k>0} c_{k} \varphi_{k}, \text { with } \sum_{k>0}\left|c_{k}\right|^{2} \ell_{k}<\infty\right\} \\
& +\left\{f \in H^{-1 / 2}\left(\Sigma, \mathbb{E}_{\mid \Sigma}\right) \mid f=\sum_{k<0} c_{k} \varphi_{k}, \text { with } \sum_{k<0}\left|c_{k}\right|^{2}\left|\ell_{k}\right|^{-1}<\infty\right\} \\
& =\theta_{+}^{-}+\theta_{-}^{-} .
\end{aligned}
$$

For the determination of the space $\beta^{-}$see [Ho1] and [APS].

Remark 2.4. By the conditions (a1) and (a2) we know that $\operatorname{Ker}\left(B_{0}\right)$ is a finite dimensional symplectic subspace of $\beta^{-}$, so that we choose eingensections $\left\{\varphi_{k}\right\}$ for $0<|k| \leq N_{0}$ in such a way that the subspaces spanned by $\left\{\varphi_{k}\right\}_{-N_{0} \leq k<0}$ and $\left\{\varphi_{k}\right\}_{0<k \leq N_{0}}$ are mutually transversal Lagrangian subspaces in $\operatorname{Ker}\left(B_{0}\right)$.

Remark 2.5. Of course for smooth sections of $\mathbb{E}_{\mid \Sigma}$ (and also for $L_{2}$-sections) the symplectic structure defined by $\sigma$ coincides with $\omega$ defined in (2.7).

Let $\gamma^{-}: \mathfrak{D}_{\mathbf{M}}^{-} \rightarrow \beta^{-}$be the projection map, then the image $\gamma^{-}\left(\operatorname{Ker}\left(\mathcal{A}_{-}^{*}\right)\right)$ is a Lagrangian subspace and the pairs $\left(\gamma^{-}\left(\operatorname{Ker}\left(\mathcal{A}_{-}^{*}\right)\right), \gamma^{-}\left(\mathfrak{D}_{A P S}^{0}\right)\right)$ and $\left(\gamma^{-}\left(\operatorname{Ker}\left(\mathcal{A}_{-}^{*}\right)\right), \gamma^{-}\left(\mathfrak{D}_{0}\right)\right)$ are Fredholm pairs. The spaces $\gamma^{-}\left(\operatorname{Ker}\left(\mathcal{A}_{-}^{*}\right)\right)$ is called Cauchy data space. Further, for each Lagrangian subspace $\lambda \subset \beta^{-}$the operator $\left.\mathcal{A}_{-}^{*}\right|_{\left(\gamma^{-}\right)^{-1}(\lambda)}$ is a selfadjoint realization and if $\left(\lambda, \gamma^{-}\left(\operatorname{Ker}\left(\mathcal{A}_{-}^{*}\right)\right)\right)$ is a Fredholm pair, then $\left.\mathcal{A}_{-}^{*}\right|_{\left(\gamma^{-}\right)^{-1}(\lambda)}$ is a selfadjoint Fredholm operator.

Remark 2.6. Note that the Lagrangian property of the Cauchy data space $\gamma^{-}\left(\operatorname{Ker}\left(\mathcal{A}_{-}^{*}\right)\right)$ would not be trivial. To prove this property we rely on the existence of at least one selfadjoint realization of $A_{\mid \mathfrak{D}_{\mathbf{m}}^{-}}$(= restriction of $\mathcal{A}_{-}^{*}$ to a suitable subspace in $\left.\mathfrak{D}_{\mathbf{M}}^{-}\right)$with compact resolvents (or a unbounded selfadjoint Fredholm extension). For our case this realization is given by the operator $A_{\mathfrak{D}_{A P S}^{0}}$, and for which proof we use the assumption (a2). 
We denote by $\mathcal{F} \Lambda_{\lambda}\left(\beta^{-}\right)$the space of Lagrangian subspaces $\mu$ in $\beta^{-}$such that $(\mu, \lambda)$ is a Fredholm pair:

$\mathcal{F} \Lambda_{\lambda}\left(\beta^{-}\right)=\left\{\mu \subset \beta^{-} \mid \mu\right.$ is a Lagrangian subspace and $(\mu, \lambda)$ is a Fredholm pair $\}$.

Now we have

\section{Theorem 2.7.}

$$
\mathcal{F} \Lambda_{\gamma^{-}\left(\mathfrak{D}_{0}\right)}\left(\beta^{-}\right)=\mathcal{F} \Lambda_{\gamma^{-}\left(\mathfrak{D}_{A P S}^{0}\right)}\left(\beta^{-}\right),
$$

more precisely the orthogonal projection operators onto the subspace $\gamma^{-}\left(\mathfrak{D}_{0}\right)$ and that onto the subspace $\gamma^{-}\left(\mathfrak{D}_{A P S}^{0}\right)=\theta_{+}^{-}$differ by a compact operator.

We prove this in the next section.

Remark 2.8. Let $H$ be a symplectic Hilbert space and we regard $H$ as a complexification of a Lagrangian subspace $\lambda$. Then a Lagrangian subspace $\mu$ is of the form $U(\lambda)=\mu$ with a unitary operator $U$ of the form Id+ compact operator, then $\mathcal{F} \Lambda_{\lambda}(H)=\mathcal{F} \Lambda_{\mu}(H)$. Also this property is equivalent to the condition that the difference of the orthogonal projection operators onto the Lagrangian subspaces $\lambda$ and $\mu$ is compact. For such two Lagrangian subspaces $\lambda$ and $\mu$ and two arbitrary Lagrangian subspaces $\nu_{0}$ and $\nu_{1}$ in $\mathcal{F} \Lambda_{\lambda}(H)=\mathcal{F} \Lambda_{\mu}(H)$ we have a well-defined integer $\sigma\left(\nu_{0}, \nu_{1} ; \lambda, \mu\right)$, called Hörmander index. This is the difference of the Maslov indexes

$$
\sigma_{H}\left(\nu_{0}, \nu_{1} ; \lambda, \mu\right)=\operatorname{Mas}(\{c(t)\}, \lambda)-\operatorname{Mas}(\{c(t)\}, \mu),
$$

where the path $\{c(t)\}$ is in $\mathcal{F} \Lambda_{\lambda}(H)$ connecting $\nu_{0}$ and $\nu_{1}$ and the difference does not depend on any such paths. Here the Maslov index $\operatorname{Mas}\left(\left\{c_{t}\right\}, \lambda\right)$ is , in a sense, the intersection number with the "Maslov cycle" $\mathfrak{M}_{\lambda}=\left\{\mu \in \mathcal{F} \Lambda_{\lambda}(H) \mid \mu \cap \lambda \neq\{0\}\right\}$ ([Ho2], $[\mathrm{Fu}$ ).

\section{Symplectic Reduction theOREM}

In this section after recalling a symplectic reduction theorem $([\mathrm{Fu}])$ we prove Theorem (2.7).

Let $\left(\mathcal{B}, \omega_{\mathcal{B}}\right)$ and $\left(L, \omega_{L}\right)$ be two symplectic Hilbert spaces $\left(\omega_{\mathcal{B}}\right.$ is the symplectic form and so on) with decompositions by Lagrangian subspaces $\theta_{-}, \theta_{+}, L_{-}$and $L_{+}$(Polarized symplectic Hilbert space):

$$
\mathcal{B}=\theta_{-}+\theta_{+}, L=L_{-}+L_{+}
$$

We assume that there are continuous injective maps $\mathbf{i}_{+}: L_{+} \rightarrow \theta_{+}$and $\mathbf{i}_{-}: \theta_{-} \rightarrow L_{-}$ having dense images such that

$$
\omega_{\mathcal{B}}\left(\mathbf{i}_{+}(a), x\right)=\omega_{L}\left(a, \mathbf{i}_{-}(x)\right) \text { for any } x \in \theta_{-} \text {and } a \in L_{+} .
$$

Then

Proposition 3.1. ([Fu] $)$ There is a continuous map $\tau: \mathcal{F} \Lambda_{\theta_{-}}(\mathcal{B}) \rightarrow \mathcal{F} \Lambda_{L_{-}}(L)$ such that for any continuous curve $\{c(t)\}_{t \in[0,1]}$ in $\mathcal{F} \Lambda_{\theta_{-}}(\mathcal{B})$

$$
\operatorname{Mas}\left(\{c(t)\}, \theta_{-}\right)=\operatorname{Mas}\left(\{\tau(c(t))\}, L_{-}\right) .
$$


The map $\tau$ is defined in the following way:

$$
\tau(\nu)=\left\{b+a \in L=L_{+}+\left.L_{-}\right|^{\exists} x \in \theta_{-} \text {such that } \mathbf{i}_{+}(b)+x \in \nu \text { and } a=\mathbf{i}_{-}(x)\right\} .
$$

For any decomposition of $\theta_{-}=F+F^{\prime}$ by closed subspaces $F(\operatorname{dim} F<+\infty)$ and $F^{\prime}$, we can decompose $L_{+}$by closed subspaces in such a way that $L_{+}=G+G^{\prime}$ with $\operatorname{dim} G=\operatorname{dim} F$ and $G+\mathbf{i}_{-}(F)$ is a symplectic subspace in $L$. Also in this case the subspace $F+\mathbf{i}_{+}(G)$ is a symplectic subspace in $\mathcal{B}$. Moreover the subspaces $F+\overline{\mathbf{i}_{+}\left(G^{\prime}\right)}$ and $G+\overline{\mathbf{i}_{-}\left(F^{\prime}\right)}$ are Lagrangian subspaces.

Then by replacing $\theta_{-}$with $F^{\prime}+\mathbf{i}_{+}(G), \theta_{+}$with $F+\overline{\mathbf{i}_{+}\left(G^{\prime}\right)}, L_{-}$with $G+\overline{\mathbf{i}_{+}\left(F^{\prime}\right)}$ and $L_{+}$ with $\mathbf{i}_{-}(F)+G^{\prime}$ and also by replacing the maps $\mathbf{i}_{ \pm}$in an obvious way we have a similar situation as in (3.1) and (3.2). We shall denote these new maps by $\widetilde{\mathbf{i}}_{ \pm}$, although the resulting maps $\tau$ between Fredholm-Lagrangian-Grassmannians $\mathcal{F} \Lambda_{\theta_{-}}(\mathcal{B})=\mathcal{F} \Lambda_{F^{\prime}+\mathbf{i}_{+}(G)}(\mathcal{B})$ and $\mathcal{F} \Lambda_{L_{-}}(L)=\mathcal{F} \Lambda_{G+\overline{\mathbf{i}_{-}\left(F^{\prime}\right)}}(L)$ coincides.

Note that the arguments above are guaranteed that the spaces $\mathcal{B}$ and $L$ are Hilbert spaces (see [KS] for symplectic Banach spaces).

We apply this proposition to the case $\mathcal{B}=\beta^{+}=\theta_{+}^{+}+\theta_{-}^{+}$and $L=\beta^{-}=\theta_{+}^{-}+\theta_{-}^{-}$. Note that the space $\beta^{+}$is defined as follows:

$$
\begin{aligned}
& \beta^{+}=\left\{f \in H^{-1 / 2}\left(\Sigma, \mathbb{E}_{\mid \Sigma}\right) \mid f=\sum_{k \in \mathbb{Z} \backslash\{0\}} c_{k} \varphi_{k}, \text { with } \sum_{k>0}\left|c_{k}\right|^{2} \ell_{k}^{-1}<\infty\right. \text { and } \\
& \left.\sum_{k<0}\left|c_{k}\right|^{2}\left|\ell_{k}\right|<\infty\right\} \\
& =\left\{f \in H^{-1 / 2}\left(\Sigma, \mathbb{E}_{\mid \Sigma}\right) \mid f=\sum_{k>0} c_{k} \varphi_{k}, \text { with } \sum_{k>0}\left|c_{k}\right|^{2} \ell_{k}^{-1}<\infty\right\} \\
& +\left\{f \in H^{1 / 2}\left(\Sigma, \mathbb{E}_{\mid \Sigma}\right) \mid f=\sum_{k<0} c_{k} \varphi_{k}, \text { with } \sum_{k<0}\left|c_{k}\right|^{2}\left|\ell_{k}\right|<\infty\right\} \\
& =\theta_{+}^{+}+\theta_{-}^{+} .
\end{aligned}
$$

The maps $\mathbf{i}_{ \pm}$here are given by inclusion maps.

Since $\left(\gamma^{+}\left(\operatorname{Ker}\left(\mathcal{A}_{+}^{*}\right)\right), \theta_{-}^{+}\right)$is a Fredholm pair, we can find a finite dimensional subspace $F$ in $\theta_{-}^{+}$and a corresponding finite dimensional subspace $G$ in $\theta_{+}^{-}$such that we have decompositions

$$
\theta_{-}^{+}=F+F^{\prime}, \quad \theta_{+}^{-}=G+G^{\prime}
$$

with suitable closed subspaces $F^{\prime}$ and $G^{\prime}$ and that

$$
F^{\prime}+\mathbf{i}_{+}(G) \text { and } \gamma^{+}\left(\operatorname{Ker}\left(\mathcal{A}_{+}^{*}\right)\right) \text { are transversal. }
$$

When we put $F^{\prime}+\mathbf{i}_{+}(G)=\lambda_{-}$and $F+\overline{\mathbf{i}_{+}\left(G^{\prime}\right)}=\lambda_{+}$we have a decomposition $\beta^{+}=$ $\lambda_{+}+\lambda_{-}$with Lagrangian subspaces $\lambda_{ \pm}$and the Cauchy data space is expressed as a graph of a continuous map $\mathfrak{K}: \lambda_{+} \rightarrow \lambda_{-}$. Then for such a Lagrangian subspace we have $\tau\left(\gamma^{+}\left(\operatorname{Ker}\left(\mathcal{A}_{+}^{*}\right)\right)\right)$ is the graph of the map $\widetilde{\mathbf{i}}_{-} \circ \mathfrak{K} \circ \widetilde{\mathbf{i}}_{+}$. Note here the maps $\widetilde{\mathbf{i}}_{ \pm}$should be defined in a suitable way according to the choices of the subspace $F$ and $G$ (for example, $\widetilde{\mathbf{i}}_{+}$is defined as $\mathbf{i}_{+}$on $F^{\prime}$ and $\mathbf{i}_{-}^{-1}$ on $\mathbf{i}_{-}(G)$ ).

Now the original maps $\mathbf{i}_{+}: \theta_{+}^{-} \rightarrow \theta_{+}^{+}$and $\mathbf{i}_{-}: \theta_{-}^{+} \rightarrow \theta_{-}^{-}$are compact operators by Rellich's Theorem and so the new maps $\widetilde{\mathbf{i}}_{ \pm}$are also compact. 
Let us denote the orthogonal projection operator to a closed subspace $E$ by $\mathcal{P}_{E}$. Then the difference

$$
\mathcal{P}_{\mathbf{i}_{+}(F)+G^{\prime}}-\mathcal{P}_{\tau\left(\operatorname{Ker}\left(\mathcal{A}_{+}^{*}\right)\right)}
$$

is a compact operator and the difference

$$
\mathcal{P}_{\mathbf{i}_{+}(F)+G^{\prime}}-\mathcal{P}_{\theta_{+}^{-}}
$$

is a finite rank operator.

By the definition of the map $\tau$ we have $\gamma^{-}\left(\mathfrak{D}_{0}\right)=\tau\left(\gamma^{+}\left(\operatorname{Ker}\left(\mathcal{A}_{+}^{*}\right)\right)\right)$, and (3.3) and (3.4) imply that the difference of the orthogonal projection operators onto the subspaces $\gamma^{-}\left(\mathfrak{D}_{A P S}^{0}\right)=\theta_{+}^{-}$and $\gamma^{-}\left(\mathfrak{D}_{0}\right)$ is a compact operator.

So this gives us a proof of Theorem 2.7).

\section{Cauchy Data spaces And Hörmander indeX}

Let $L_{2}(\Sigma)=L_{+}+L_{-}$be the polarization by $L_{ \pm}$, where $L_{+}$is the $L_{2}$-completion of the space spanned by $\left\{\varphi_{k}\right\}_{k>0}$ and $L_{-}$is the $L_{2}$-completion of the space spanned by $\left\{\varphi_{k}\right\}_{k<0}$. Then by applying above arguments to the two pairs $\left(\beta^{+}=\beta_{-}^{+}+\beta_{+}^{+}, L_{2}(\Sigma)=L_{-}+L_{+}\right)$ and $\left(\beta^{-}=\beta_{-}^{-}+\beta_{+}^{-}, L_{2}(\Sigma)=L_{-}+L_{+}\right)$of polarized symplectic Hilbert spaces we have four Lagrangian subspaces

$$
\gamma^{ \pm}\left(\operatorname{Ker}\left(\mathcal{A}_{ \pm}^{*}\right)\right) \cap L_{2}(\Sigma), L_{ \pm}
$$

of $L_{2}(\Sigma)$ which satisfy following properties (h1) and (h2):

(h1) $: \gamma^{ \pm}\left(\operatorname{Ker}\left(\mathcal{A}_{ \pm}^{*}\right)\right) \cap L_{2}(\Sigma)$ and $L_{\mp}$ are Fredholm pairs, $(\mathbf{h} 2): \gamma^{ \pm}\left(\operatorname{Ker}\left(\mathcal{A}_{ \pm}^{*}\right)\right) \cap L_{2}(\Sigma)=U_{ \pm}\left(L_{ \pm}\right)$, where $U_{ \pm}$are unitary operators of the form Id + compact operator.

Here we identify $L_{2}(\Sigma) \cong L_{+} \otimes \mathbb{C}$.

Now we can define the Hörmander index

$$
\sigma_{H}\left(\gamma^{+}\left(\operatorname{Ker}\left(\mathcal{A}_{+}^{*}\right)\right) \cap L_{2}(\Sigma), L_{+} ; \gamma^{-}\left(\operatorname{Ker}\left(\mathcal{A}_{-}^{*}\right)\right) \cap L_{2}(\Sigma), L_{-}\right)
$$

of these four Lagrangian subspaces. Then its absolute value will express an asymmetry of solution spaces of the operator $A$ under the decomposition of $M$ along a hypersurface $\Sigma$. So, if there is a symmetry among these four Lagrangian subspaces, the value must vanish. In fact

Proposition 4.1. Assume that $\sigma\left(\gamma^{+}\left(\operatorname{Ker}\left(\mathcal{A}_{+}^{*}\right)\right) \cap L_{2}(\Sigma)\right)=\gamma^{-}\left(\operatorname{Ker}\left(\mathcal{A}_{-}^{*}\right)\right) \cap L_{2}(\Sigma)$, then the Hörmander index of these four Lagrangian subspaces vanishes:

$$
\sigma_{H}\left(\gamma^{+}\left(\operatorname{Ker}\left(\mathcal{A}_{+}^{*}\right)\right) \cap L_{2}(\Sigma), L_{+} ; \gamma^{-}\left(\operatorname{Ker}\left(\mathcal{A}_{-}^{*}\right)\right) \cap L_{2}(\Sigma), L_{-}\right)=0 .
$$

Proof. First we assume that $\gamma^{+}\left(\operatorname{Ker}\left(\mathcal{A}_{+}^{*}\right)\right) \cap L_{2}(\Sigma)$ and $L_{-}$are transversal. Then the space $\gamma^{+}\left(\operatorname{Ker}\left(\mathcal{A}_{+}^{*}\right)\right) \cap L_{2}(\Sigma)$ is written as a graph of a compact operator $T: L_{+} \rightarrow L_{-}$ such that $\sigma \circ T$ is a selfadjoint operator on $L_{+}$and the space $\gamma^{-}\left(\operatorname{Ker}\left(\mathcal{A}_{-}^{*}\right)\right) \cap L_{2}(\Sigma)$ is also written as a graph of the map $-\sigma \circ T \circ \sigma$. These imply that the curve of Lagrangian subspaces given by the graphs of $\{-t \cdot \sigma \circ T \circ \sigma\}_{0 \leq t \leq 1}$ is always transversal to both of $\gamma^{+}\left(\operatorname{Ker}\left(\mathcal{A}_{+}^{*}\right)\right) \cap L_{2}(\Sigma)$ and $L_{+}$. This curve is connecting $\gamma^{-}\left(\operatorname{Ker}\left(\mathcal{A}_{-}^{*}\right)\right) \cap L_{2}(\Sigma)$ and $L_{-}$. Hence we have

$$
\sigma_{H}\left(\gamma^{-}\left(\operatorname{Ker}\left(\mathcal{A}_{-}^{*}\right)\right) \cap L_{2}(\Sigma), L_{-} ; \gamma^{+}\left(\operatorname{Ker}\left(\mathcal{A}_{+}^{*}\right)\right) \cap L_{2}(\Sigma), L_{+}\right)=0 .
$$


If $\gamma^{+}\left(\operatorname{Ker}\left(\mathcal{A}_{+}^{*}\right)\right) \cap L_{2}(\Sigma)$ and $L_{-}$are not transversal, then we decompose the Lagrangian subspace $\gamma^{+}\left(\operatorname{Ker}\left(\mathcal{A}_{+}^{*}\right)\right) \cap L_{2}(\Sigma)$ into the orthogonal sum

$$
\gamma^{+}\left(\operatorname{Ker}\left(\mathcal{A}_{+}^{*}\right)\right) \cap L_{2}(\Sigma)=\ell_{0}+\nu,
$$

where $\ell_{0}=\left(\gamma^{+}\left(\operatorname{Ker}\left(\mathcal{A}_{+}^{*}\right)\right) \cap L_{2}(\Sigma)\right) \cap L_{-}$and $\nu$ is the orthogonal complement of $\ell_{0}$ in $\gamma^{+}\left(\operatorname{Ker}\left(\mathcal{A}_{+}^{*}\right)\right) \cap L_{2}(\Sigma)$. Also we decompose $L_{-}=\ell_{0}+\left(L_{-} \cap \ell_{0}^{\perp}\right)=\ell_{0}+\ell_{-}$and $L_{+}=$ $\sigma\left(\ell_{0}\right)+\left(L_{+} \cap \sigma\left(\ell_{0}\right)^{\perp}\right)=\sigma\left(\ell_{0}\right)+\ell_{+}$. Now we have

$$
\begin{aligned}
& \sigma_{H}\left(\gamma^{-}\left(\operatorname{Ker}\left(\mathcal{A}_{-}^{*}\right)\right) \cap L_{2}(\Sigma), L_{-} ; \gamma^{+}\left(\operatorname{Ker}\left(\mathcal{A}_{+}^{*}\right)\right) \cap L_{2}(\Sigma), L_{+}\right) \\
& =\sigma_{H}\left(\sigma\left(\ell_{0}\right), \ell_{0} ; \ell_{0}, \sigma\left(\ell_{0}\right)\right)+\sigma_{H}\left(\sigma(\nu), \ell_{-} ; \nu, \ell_{+}\right)=0,
\end{aligned}
$$

by applying the first arguments to the second term.

Note that $\gamma^{-}\left(\operatorname{Ker}\left(\mathcal{A}_{-}^{*}\right)\right) \cap L_{2}(\Sigma)=\sigma\left(\ell_{0}\right)+\sigma(\nu)$ is an orthogonal decomposition and the vanishing of the first term follows from a skew-symmetric property of the Hörmander index.

\section{A splitting Formula of a spectral Flow}

First we state a splitting formula for a spectral flow when we decompose a manifold into two components. Then we give another form of it by replacing the boundary condition with Atiyah-Patodi-Singer condition.

Let $\left\{C_{t}\right\}_{t \in[0,1]}$ be a continuous family of symmetric bundle maps of $\mathbb{E}$ and we assume that each of the operator in the family $\left\{A+C_{t}\right\}$ satisfies the conditions (a1') and (a2) where (a1') is:

(a1'): There exists an $\epsilon_{0}>0$ such that for any $|s|<\epsilon_{0}$ and any $t \in[0,1]$ the operators $A+C_{t}+s$ satisfy the unique continuation property with respect to the hypersurface $\Sigma$ :

$$
\operatorname{Ker}\left(\mathcal{A}_{ \pm}^{*}+C_{t}+s\right) \cap \mathfrak{D}_{\mathbf{m}}^{ \pm}=\{0\} .
$$

Here $C_{t}$ is regarded as a bounded selfadjoint operator on $L_{2}(M, \mathbb{E})$.

Now we have continuous families of Cauchy data spaces $\gamma^{ \pm}\left(\operatorname{Ker}\left(\mathcal{A}_{ \pm}^{*}+C_{t}\right)\right)\left(C_{t}\right.$ should be considered as acting on the space $\mathfrak{D}_{\mathbf{M}}^{ \pm}$respectively, and both of which are invariant under this action). The splitting formula is stated as follows:

Theorem 5.1. ([FO])

$$
\mathbf{S f}\left(\left\{A+C_{t}\right\}\right)=\mathbf{S f}\left(\left\{A_{\mathfrak{D}_{0}}+C_{t}\right\}\right)+\mathbf{S f}\left(\left\{A_{\mathfrak{D}_{1}}+C_{t}\right\}\right),
$$

where

$\mathfrak{D}_{0}=\left\{\left.f \in H^{1}\left(M_{-}, \mathbb{E}_{\mid M_{-}}\right)\right|^{\exists} \widetilde{f} \in H^{1}(M, \mathbb{E})\right.$ such that $\widetilde{f}_{\mid M_{-}}=f$ and $\left(A+C_{0}\right)(\widetilde{f})=0$ on $\left.M_{+}\right\}$ and

$\mathfrak{D}_{1}=\left\{\left.g \in H^{1}\left(M_{+}, \mathbb{E}_{\mid M_{+}}\right)\right|^{\exists} \widetilde{g} \in H^{1}(M, \mathbb{E})\right.$ such that $\widetilde{g}_{\mid M_{+}}=g$ and $\left(A+C_{1}\right)(\widetilde{g})=0$ on $\left.M_{-}\right\}$.

Remark 5.2. Our proof of the general spectral flow formula bases on the property (a1') and (a2), and by making use of the general spectral flow formula and $L_{2}$ reduction theorem we prove the splitting formula above $([\mathrm{FO}])$. 
Let $\mathfrak{D}_{A P S}^{0}$ be the space defined in (2.6) for $A$ replaced by $A+C_{0}$ and denote by $\mathfrak{D}_{A P S}^{1}$ the similar space

$$
\begin{aligned}
& \mathfrak{D}_{A P S}^{1} \\
& =\left\{f \in H^{1}\left(M_{+}, \mathbb{E}_{\mid M_{+}}\right) \mid \text {if } f_{\mid \Sigma}=\sum_{k \in \mathbb{Z} \backslash\{0\}} a_{k} \psi_{k} \text {, then } a_{k}=0 \text { for } k=1,2,3, \cdots\right\} .
\end{aligned}
$$

Note that the sections $\left\{\psi_{k}\right\}$ are now orthonormal eigensections of the tangential operator $B_{1}$ in the product form

$$
A+C_{1}=\sigma\left(\frac{\partial}{\partial u}+B_{1}\right)
$$

corresponding to the parameter $t=1$ and should be chosen in such a way as noted in Remark (2.4).

We have continuous curves $\left\{\gamma^{-}\left(\operatorname{Ker}\left(\mathcal{A}_{-}^{*}+C_{t}\right)\right)\right\}$ of Cauchy data spaces in the FredholmLagrangian-Grassmannian $\mathcal{F} \Lambda_{\mathfrak{D}_{0}}\left(\beta^{-}\right)=\mathcal{F} \Lambda_{\mathfrak{D}_{A P S}^{0}}\left(\beta^{-}\right)$and $\left\{\gamma^{+}\left(\operatorname{Ker}\left(\mathcal{A}_{+}^{*}+C_{t}\right)\right)\right\}$ in $\mathcal{F} \Lambda_{\mathfrak{D}_{1}}\left(\beta^{+}\right)=\mathcal{F} \Lambda_{\mathfrak{D}_{A P S}^{1}}\left(\beta^{+}\right)$.

The Hörmander index is defined for four Lagrangian subspaces $\mu_{0}, \mu_{1}, \gamma^{-}\left(\mathfrak{D}_{0}\right)$ and $\gamma^{-}\left(\mathfrak{D}_{A P S}^{0}\right)$, where $\mu_{i} \in \mathcal{F} \Lambda_{\mathfrak{D}_{0}}\left(\beta^{-}\right)=\mathcal{F} \Lambda_{\mathfrak{D}_{A P S}^{0}}\left(\beta^{-}\right)$, also defined for $\nu_{0}, \nu_{1}, \gamma^{+}\left(\mathfrak{D}_{1}\right)$ and $\gamma^{+}\left(\mathfrak{D}_{A P S}^{1}\right)\left(\nu_{i} \in \mathcal{F} \Lambda_{\mathfrak{D}_{1}}\left(\beta^{+}\right)=\mathcal{F} \Lambda_{\mathfrak{D}_{A P S}^{1}}\left(\beta^{+}\right)\right)$, as noted in Remark (2.8).

Since

$$
\begin{aligned}
\operatorname{Sf}\left(\left\{A_{\mathfrak{D}_{0}}+C_{t}\right\}\right) \\
=\operatorname{Mas}\left(\left\{\gamma^{-}\left(\operatorname{Ker}\left(\mathcal{A}_{-}^{*}+C_{t}\right)\right)\right\}, \gamma^{-}\left(\mathfrak{D}_{0}\right)\right) \\
=\operatorname{Mas}\left(\left\{\gamma^{-}\left(\operatorname{Ker}\left(\mathcal{A}_{-}^{*}+C_{t}\right)\right)\right\}, \gamma^{-}\left(\mathfrak{D}_{A P S}^{0}\right)\right) \\
\quad+\sigma_{H}\left(\gamma^{-}\left(\operatorname{Ker}\left(\mathcal{A}_{-}^{*}+C_{0}\right)\right), \gamma^{-}\left(\operatorname{Ker}\left(\mathcal{A}_{-}^{*}+C_{1}\right)\right) ; \gamma^{-}\left(\mathfrak{D}_{0}\right), \gamma^{-}\left(\mathfrak{D}_{A P S}^{0}\right)\right)
\end{aligned}
$$

we have

\section{Theorem 5.3.}

$$
\begin{aligned}
& \mathbf{S f}\left(\left\{A+C_{t}\right\}\right) \\
& =\operatorname{Mas}\left(\left\{\gamma^{-}\left(\operatorname{Ker}\left(\mathcal{A}_{-}^{*}+C_{t}\right)\right)\right\}, \gamma^{-}\left(\mathfrak{D}_{A P S}^{0}\right)\right) \\
& +\sigma_{H}\left(\gamma^{-}\left(\operatorname{Ker}\left(\mathcal{A}_{-}^{*}+C_{0}\right)\right), \gamma^{-}\left(\operatorname{Ker}\left(\mathcal{A}_{-}^{*}+C_{1}\right)\right) ; \gamma^{-}\left(\mathfrak{D}_{0}\right), \gamma^{-}\left(\mathfrak{D}_{A P S}^{0}\right)\right) \\
& +\operatorname{Mas}\left(\left\{\gamma^{+}\left(\operatorname{Ker}\left(\mathcal{A}_{+}{ }^{*}+C_{t}\right)\right)\right\}, \gamma^{+}\left(\mathfrak{D}_{A P S}^{1}\right)\right) \\
& +\sigma_{H}\left(\gamma^{+}\left(\operatorname{Ker}\left(\mathcal{A}_{+}^{*}+C_{0}\right)\right), \gamma^{+}\left(\operatorname{Ker}\left(\mathcal{A}_{+}^{*}+C_{1}\right)\right) ; \gamma^{+}\left(\mathfrak{D}_{1}\right), \gamma^{+}\left(\mathfrak{D}_{A P S}^{1}\right)\right) \\
& =\mathbf{S f}\left(\left\{A_{\mathfrak{D}_{A P S}^{0}}+C_{t}\right\}\right)+\mathbf{S f}\left(\left\{A_{\mathfrak{D}_{A P S}^{1}}+C_{t}\right\}\right) \\
& +\sigma_{H}\left(\gamma^{-}\left(\operatorname{Ker}\left(\mathcal{A}_{-}^{*}+C_{0}\right)\right), \gamma^{-}\left(\operatorname{Ker}\left(\mathcal{A}_{-}^{*}+C_{1}\right)\right) ; \gamma^{-}\left(\mathfrak{D}_{0}\right), \gamma^{-}\left(\mathfrak{D}_{A P S}^{0}\right)\right) \\
& +\sigma_{H}\left(\gamma^{+}\left(\operatorname{Ker}\left(\mathcal{A}_{+}^{*}+C_{0}\right)\right), \gamma^{+}\left(\operatorname{Ker}\left(\mathcal{A}_{+}^{*}+C_{1}\right)\right) ; \gamma^{+}\left(\mathfrak{D}_{1}\right), \gamma^{+}\left(\mathfrak{D}_{A P S}^{1}\right)\right) \text {. }
\end{aligned}
$$

Corollary 5.4. If the family $\left\{A+C_{t}\right\}$ is a loop, i.e., $C_{0}=C_{1}$, then we have

$$
\mathbf{S f}\left(\left\{A+C_{t}\right\}\right)=\mathbf{S f}\left(\left\{A_{\mathfrak{D}_{A P S}^{0}}+C_{t}\right\}\right)+\mathbf{S f}\left(\left\{A_{\mathfrak{D}_{A P S}^{1}}+C_{t}\right\}\right) .
$$

Remark 5.5. Although it holds the spectral flow formula expressed in terms of the Maslov index of Cauchy data spaces under the assumption (a1'), it would not be clear whether the splitting formulas of the spectral flow like above formulas hold always without the second assumption (a2). Such assumptions are fit to the framework of the symplectic 
Hilbert space theory, after once the spaces $\beta^{ \pm}$are determined. However it would be expected that generalizations of splitting formula of spectral flow and the index similar to (4.1) without the assumption (a2) would be carried out through a further analysis of the pseudo-differential operator theory including the Calderón projector and the operator $\mathcal{P}_{\tau\left(\operatorname{Ker}\left(\mathcal{A}_{+}^{*}\right)\right)}$.

\section{REFERENCES}

[APS] M. F. Atiyah, V.K. Patodi and I. M. Singer, Spectral asymmetry and Riemannian geometry: I, Math. Proc. Camb. Phil. Soc., 77(1975), 43-69. II, Math. Proc. Camb. Phil. Soc., 78(1975), 405-432. III, Math. Proc. Camb. Phil. Soc., 79(1976), 71-99.

[ClM1] S.E. Cappell, R. Lee, and E.Y. Miller, On the Maslov index, Comm. Pure Appl. Math. 47 (1994), 121-186.

[CLM2] - - - - - Selfadjoint elliptic operators and manifold decompositions Part I: Low eigenmodes and stretching, Comm. Pure Appl. Math. 49 (1996), 825-866. Part II: Spectral flow and Maslov index, Comm. Pure Appl. Math. 49 (1996), 869-909. Part III: Determinant line bundles and Lagrangian intersection, Comm. Pure Appl. Math. 52 (1999), 543-611.

[DK] M. Daniel and P. Kirk, with an appendix By K.P. Wojciechowski, A general splitting formula for the spectral flow, Michigan Math. Journal 46(1999), 589-617.

[FO] K. Furutani and N. Otsuki, Maslov index in the infinite dimension and a splitting formula for a spectral flow, Japanese Journal of Mathathematics. Vol. 28, No. 2(2002), 215-243.

[Fu] K. Furutani, Fredholm-Lagrangian-Grassmannian and the Maslov index, Journal of Geometry and Physics, to appear.

[Go] M. De Gosson, La définition de l'indice de Maslov sans hypothèse de transversalité, C. R. Acad. Sci. Paris 310, Série I (1990), 279-282.

[Ho1] L. Hoermander, Pseudo-differential operators and non-elliptic boundary problems, Ann. of Math. 83 (1966), 129-209.

[Ho2] —, Fourier integral operators I, Acta Math, 127(1971), 79-183.

[KL] P. KiRK And M. LESCH, The $\eta$-invariant, Maslov index, and spectral flow for Dirac-type operators on manifolds with boundary, (preprint).

[KS] N. J. Kalton and R. C. Swanson, A symplectic Banach space with no Lagrangian subspaces, Transaction of the Amer. Math. Soc.,273, No 1 (1982), 385-392.

[Ni] L. Nicolaescu, The Maslov index, the spectral flow, and decomposition of manifolds, Duke Math. J. 80 (1995), 485-533.

[RS] J. Robbin and D. Salamon, The Maslov index for paths, Topology 32 (1993), 827-844.

[Ta] C. H. Taubes, Casson's invariant and gauge theory, J. Differential Geometry 31(1990), 547-599.

[Yo] T. YoshidA, Floer homology and splittings of manifolds, Ann. of Math. 134 (1991), 277-323.

Kenro Furutani

Department of Mathematics

Faculty of Science and Technology

Science University of Tokyo

2641 Noda, Chiba (278-8510)

JAPAN

E-mail address: furutani@ma.noda.sut.ac.jp 